\title{
Intermittency studies in directed bond percolation
}

\author{
Malte Henkel ${ }^{a}$ 的 Robert Peschanski ${ }^{b}$ \\ ${ }^{a}$ Département de Physique Théorique, Université de Genève \\ 24 quai Ernest Ansermet, CH - 1211 Genève 4, Switzerland \\ ${ }^{b}$ Service de Physique Théoriquę, CEN Saclay \\ F- 91191 Gif sur Yvette Cedex, France
}

UGVA-DPT 1992/06-769

Saclay SPhT/92-069

\begin{abstract}
The self-similar cluster fluctuations of directed bond percolation at the percolation threshold are studied using techniques borrowed from intermittency-related analysis in multi-particle production. Numerical simulations based on the factorial moments for large $1+1$-dimensional lattices allow to handle statistical and boundary effects and show the existence of weak but definite intermittency patterns. The extracted fractal dimensions are in agreement with scaling arguments leading to a new relation linking the intermittency indices to the critical exponents and the fractal dimension of directed percolation clusters.
\end{abstract}

\footnotetext{
${ }^{1}$ Supported in part by the Swiss National Science Foundation

${ }^{2}$ Laboratoire de la Direction des Sciences de la Matière du Commissariat à l'Energie Atomique
} 


\section{Introduction}

The transition between integrable and chaotic behaviour has attracted interest for quite a long time. In particular, in the context of hydrodynamics, the spatio-temporal intermittent behaviour has been studied [1]. On the other hand, similar structures have shown up in the context of particle fragmentation at high energies, as first exemplified by the JACEE cosmic-ray event and later on by many studies at particle accelerators [2]. Intermittency studies have also been performed in Statistical Mechanics, on systems like the $2 D$ Ising model at the second-order transition [3, 4. In this work, we consider directed bond percolation as a simple toy model for intermittent behaviour. As we shall argue below, it might provide a convenient model approach to describe some basic properties of intermittent systems.

Our interest in studying intermittency was triggered by the observation that directed percolation can be used as a simple non-perturbative model for particle fragmentation as described by the Schwinger mechanism [5, 6]. Already before that, it had been proposed that directed percolation might be useful in describing the transition between laminar and turbulent flow, occuring for example in hydrodynamics [1]. We shall describe these heuristic arguments in section 2. For the quantitative description of intermittent behaviour, a practical tool are the factorial moments [7]

$$
F_{q}:=\frac{<n(n-1) \cdots(n-q+1)>}{<n>^{q}}
$$

where $n$ is the number of particles in a certain (rapidity) interval and $<>$ denotes the average over a given sample. We shall recall some of their properties in section 2. Intermittent behaviour means that the $F_{q}$ obey a power law as a function of the binning size $\delta$

$$
F_{q}(\delta) \sim \delta^{-\phi_{q}}
$$

which defines the intermittency indices $\phi_{q}$. The verification of this relation and the eventual calculation of these numbers will be the first objective of this paper, to be described in section 3 . From the $\phi_{q}$, one obtains the Renyi codimension $\overline{\mathcal{D}}_{q}$

$$
\overline{\mathcal{D}}_{q}=\phi_{q} /(q-1)
$$

If the fluctuations of the model have a fractal nature, the $\overline{\mathcal{D}}_{q}$ will be $q$ independent, while for a multifractal, the $\overline{\mathcal{D}}_{q}$ retain a dependence on $q$. In 
the case of a simple fractal behaviour, when $\overline{\mathcal{D}}_{q}=\overline{\mathcal{D}}$ for all $q$, we shall obtain a relationship with the fractal codimension $\bar{D}$ of the directed percolation cluster and critical exponents (see section 4)

$$
\overline{\mathcal{D}}=\beta / \nu_{\perp}-\bar{D} \geq 0
$$

This result allows to reconsider the recent comparison between numerical calculations of fractal cluster dimensions with theoretical ideas [8]. It also casts a bridge with the Reggeon Field Theory, which furnishes a continuum field theory description of directed percolation [9]. The validity of the result (1.4) only depends on simple scaling arguments and should therefore not be restricted to critical directed percolation systems. Section 5 presents our conclusions.

\section{Directed percolation and intermittency}

\subsection{Some heuristics}

We recall the definition of directed percolation (see e.g. [10]). Consider a lattice and single out a preferred direction. This preferred direction will be called "time", the other ones are referred to as "space". For the purposes of this paper, we shall limit ourselves exclusively to the case of one temporal and one spatial dimension. The sites of the lattice can be either filled (1) with probability $\tilde{p}$ or empty (0) with probability $1-\tilde{p}$. A bond between sites is present with probability $p$ and absent with probability $1-p$. The occupation $v_{i, t}$ of the sites is defined in terms of the conditional probabilities $P\left(v_{i, t+1} \mid v_{i-1, t}, v_{i+1, t}\right)$, see fig. 1, namely,

$$
P(1 \mid 0,0)=0, P(1 \mid 1,1)=p(2-p) \tilde{p}, \quad P(1 \mid 0,1)=P(1 \mid 1,0)=p \tilde{p}
$$

together with $P\left(0 \mid v, v^{\prime}\right)=1-P\left(1 \mid v, v^{\prime}\right)$. For $\tilde{p}=1$, one has directed bond percolation and for $p=1$ one has directed site percolation. The probability

$\mathcal{P}$ for having an infinite directed cluster of connected sites scales with the distance from the percolation threshold $p_{c}$ as $\mathcal{P} \sim\left(p-p_{c}\right)^{2 \beta}$ and has the role of an order parameter. The scaling of the temporal and spatial correlation lengths $\xi_{\|}$and $\xi_{\perp}$ is described by exponents $\nu_{\|}$and $\nu_{\perp}$, viz.

$$
\xi_{\|} \sim\left(p-p_{c}\right)^{-\nu_{\|}}, \xi_{\perp} \sim\left(p-p_{c}\right)^{-\nu_{\perp}}
$$


Numerical values are 11]

$$
\begin{array}{ll}
\tilde{p}_{c}=0.705489(4) & \text { square site } \\
p_{c}=0.644701(1) & \text { square bond }
\end{array}
$$

and for the exponents 11

$$
\beta=0.277(2), \nu_{\|}=1.7339(3), \nu_{\perp}=1.0969(3)
$$

From these, all other exponents can be found by scaling relations. For a test of universality for the exponents and the two-point correlation scaling function, see [12].

The problem of directed percolation can be rewritten in terms of a field theory. As it is well known, directed bond percolation is in the same universality class as Reggeon field theory [9], at least close to the upper critical dimension $D^{*}=5$. Nevertheless, this universality was also confirmed numerically for $D=2$ and $D=3$, see refs. [13, 14, 15]. Consequently, results of a directed percolation study also carry an implication about that theory. We will discuss that point later on.

Some time ago, Pomeau [1] has proposed that spatial-temporal intermittency were in the same universality class as directed percolation. According to him, the transition to the turbulent state in a liquid is similar to the one induced by the intermittent transition in oscillations and that the interaction between neighboring oscillators might be seen as some sort of local contamination process. It appears then plausible that an oscillator in a turbulent state may either relax spontaneously towards its quiescent state or else may contaminate its neighbors. As an example of this, one might consider the observation that the laminar convection rolls in Taylor-Couette flow do not break up spontaneously into a turbulent state but get only infected via a turbulent neighbor. This is nothing else than the familar definition of directed percolation. Although this argument is purely qualitative and does not yield a value for the percolation probability $p$, it does carry some heuristic value, even if the simple directed percolation is probably not sufficient to describe intermittency occuring in realistic liquids [16].

To complement this, we now argue that directed percolation might be useful to study some aspects of particle fragmentation. For simplicity, consider the example of $e^{+} e^{-}$annihilation producing a quark-antiquark pair. It is generally believed that for a confining theory like QCD the color lines of 
force are essentially concentrated in a narrow tube connecting $q$ with $\bar{q}$, acting like a string with a constant tension. By the Schwinger mechanism, new $q \bar{q}$ pairs are created out of the vacuum by the color force with a probability $P$ expressible in terms of known quantities [5, 6]. One thus obtains a resulting color field which will strongly fluctuate as a function of the relative rapidity between the particles.

We now make the link from directed percolation to this model. Since the color flux tube is essentially one-dimensional, we take a two-dimensional lattice. The direction perpendicular ("space") to the imposed propagation ("time") direction will be taken to correspond to the rapidity interval. On each site of the spatial lattice we put 0 if there is no color field and 1 if there is one. The quarks or antiquarks generated are placed on the dual spatial lattice. The creation of a new $q \bar{q}$ pair generates a supplementary color field which exactly compensates the one which produced the new pair. In the particle language, this means that some local field energy has been transformed in particle creation with probability $P$. In the percolation language, this corresponds to having a site occupied by 0 at time $t$ after its two neighbors at time $t-1$ have been occupied by one. In the frame of directed bond percolation, see $(2.1)$, we have $P=1-P(1 \mid 1,1)=(1-p)^{2}$. Again, this very simple model should be taken as a guide but not as a realistic description of high-energy processes.

The breaking of the color field is stochastic and, due to the causality nature of directed percolation, new particles can only be created where the field persits, it generates a cascade. Since randomness and cascading are just the two features known to give intermittency in particle production [5], and because of the connection with turbulent behaviour [1], we expect to find intermittency in the context of directed percolation. This means the occurence of short-range self-similar fluctuations which dominate the average characteristics of a multiparticle system.

This rough model description motivates our simulations of $1+1$ dimensional directed percolation for particle physics. However it is worth noting that $2+1$-dimensional directed percolation has another more theoretical relation with multi-particle collisions, thanks to its continuum limit in terms of the Reggeon Field Theory. In this context, the "time" dimension corresponds to the rapidity of produced particles in a very energetic collision, and the two-dimensional "space" is the transverse impact parameter. So, some of our results can find a prolongation in a $2+1$-dimensional study of direct 
relevance to the theory of particles.

\section{$2.2 \quad$ Factorial moments}

The factorial moment analysis [7] is useful to avoid as much as possible a "contamination by statistical noise" of the dynamical fluctuations one is looking for. The general idea is that a large part of the non-dynamical statistical fluctuations is due to the small number of particules (statistical realizations), and thus can be assimilated to a "noise" with simple stochastic characteristics. In the absence of other information, the "noise" is assumed to be Poissonian and thus can be de-convoluted from the observed distribution of particles by the factorial moments' trick. Of course, this assumption looks strong, but many model tests [7, 2] have shown its validity in multi-particle physics. On the other hand, statistical systems like directed percolation are useful to check the "noise" assumption in cases where it is embedded in the dynamics, and allow to deepen the statistical analysis.

In this specific case, however, one disposes of an information on the total number of sites under study in a given phase-space box, and thus, adding this constraint to the "noise" definition, one is led to consider the Bernoulli "noise". More specifically for lattice systems, one writes :

$$
\begin{aligned}
F_{q} & =\frac{<\rho^{q}>}{<\rho>^{q}} \\
<\rho^{q}> & =\frac{<n(n-1) \cdots(n-q+1)>}{<N(N-1) \cdots(N-q-1)>}
\end{aligned}
$$

where $N$ stands for the number of sites per bin, $\rho$ is the density of occupied sites and the average $<>$ is meant over the total number of bins. Notice that the choosen normalization for factorial moments has been designed for the Bernoulli noise [7] expected in lattice simulations [17] and checked in detailed calculations on the Ising model [18. If the factorial analysis works well, the density $\rho$ and its fluctuations are free from statistical perturbations and the intermittency parameters $\phi_{q}$ and $\overline{\mathcal{D}}_{q}$ (see (1.2), (1.3)) can be estimated.

Intermittency studies for statistical systems using factorial moments (2.4) are particularly interesting since they offer an unique opportunity to discuss a scaling behaviour of fluctuations in a well-defined dynamical scheme. There is no need of a small coupling hypothesis nor of an assumption on the statistical 
noise. Indeed, the statistical fluctuations from site to site are, together with the dynamical ones, encoded in the generic rules (2.1).

\section{Numerical simulation}

We now turn to describe our simulation procedure and the numerical results for the intermittency indices. We use directed bond percolation on a square lattice. Since an intermittent behaviour as the one in eq. (1.2) can only occur at criticality, we shall always work at the critical point $p=p_{c}$, see $(2.2)$. As the initial state, we take occupied sites (1) on all spatial sites at time $t=0$. Both periodic and free boundary conditions will be considered, see below.

The factorial moments can be calculated for a two-dimensional or a onedimensional binning. In the latter case, we can either choose a spatial (vertical) or a temporal (horizontal) one. For the calculation, one can either take subdivisions of the entire lattice with $N$ spatial sites into account or only apply the procedure of repeated subdivions to a certain subcell of $L<N$ spatial sites. Both of these procedures were tried for the case of periodic boundary conditions, while for free boundary conditions, only the second one was used. The moments were obtained calculating by generating $L$ time steps of the evolution of the directed percolation cluster. The resulting square of $L \times L$ sites was then subdivided into squares of sizes $L / 2^{b} \times L / 2^{b}$ with $b=0,1,2, \ldots$ to obtain the two-dimensional factorial moments $F_{q}^{(2 D)}$. To obtain the one-dimensional moments $F_{q}^{(H)}$ and $F_{q}^{(V)}$, in the same square for the fixed time (for $F_{q}^{(H)}$ ) or for fixed position (for $F_{q}^{(V)}$ ) subcells of length $L / 2^{b}$ were formed, see fig. 2. We take an increment of five "layers" (intermediate steps in time or space, respectively) between two consecutive calculations for the $1 D$ factorial moments with a given square of size $L \times L$. To reduce the statistical correlations between measurements, we generated 2000 "thermalisation" time evolutions steps before beginning the calculation of the factorial moments and generate an additional 2000 time steps between any two squares to be considered for measurements. Also, if the directed percolation cluster happened to die out (this only occured for fairly small lattices), we reinitialized with all spatial sites occupied and 2000 thermalisation steps. Since correlation function studies have shown that scaling sets in after about 20 thermalization steps (see [12]), this thermalization should be sufficient. Almost the entire CPU time was spent in calculating the factorial 
moments.

We now discuss the necessary spatial lattice size in order to become independent of finite-size effects. To see this, we examine the calculated factorial moments $F_{4}(\delta)$ as function of the binning size $\delta$. In fig. 3-a, we display the Renyi codimension $\overline{\mathcal{D}}_{4}$ as calculated for boxes of linear size $L / 2^{b}$ for the $2 D$ factorial moment $F_{4}^{(2 D)}$. Similarly, fig. 3-b shows the same quantity as obtained from $F_{4}^{(H)}$ and fig. 3-c those obtained from $F_{4}^{(V)}$.

We first observe that for all three cases the apparent Renyi codimensions become independent of the lattice size $N$, the size $L$ of the largest box considered and of the boundary conditions if $N$ is larger than 4096. These sizes are somewhat larger than the largest ones considered in intermittency model calculations so far (see e.g. [18]). We can conclude that the data for $N=8192$ will describe accurately the behaviour of the $N \rightarrow \infty$ limit and that finite-size effects are small for this size. Secondly, we see that, apart from the largest blocks (where $b$ is small) and the smallest one (where the cells just contains two sites), the data show a large and wide plateau and become $b$ - independent. The value of the plateau will be taken as our estimate for the Renyi codimensions. These observations hold for all the three types of factorial moments considered. We have drawn similar plots for the cases $q=2$ and $q=6$ and find the qualitative behaviour to be the same, although the convergence towards the $N \rightarrow \infty$ limit is somewhat slower for higher rank. We can conclude that our directed bond percolation model at $p=p_{c}$ does indeed show intermittent behaviour. For a precise calculation of the intermittency indices, we have seen that lattices $N=8192$ spatial sites are large enough. We have also studied the effect of varying $p$ away from $p_{c}$. Already for variations of order $\delta p \simeq 10^{-2}$, the $\overline{\mathcal{D}}_{q}$ are no longer almost independent of $b$, but are rather seen to rise steeply with increasing values of $b$.

To investigate the $q$ - dependence, we take $N=8192, L=2048$ with periodic boundary conditions. We generated 500 squares of size $L \times L$ for the calculation of the $F_{q}$, with 2000 intermediate time steps in between each square. In fig. 4-a, we give the curves for the Renyi codimension $\overline{\mathcal{D}}_{q}^{(2 D)}$, for $q=2,3,4,5,6$ as calculated from $F_{q}^{(2 D)}$. Similarly, fig. 4-b shows the results obtained from $F_{q}^{(H)}$ and fig. 4-c those from $F_{q}^{(V)}$. Already a first glance at the figures shows that the $2 D$ and the horizontal $(H)$ results are very similar to each other while the vertical $(V)$ ones are quite distinct. In both figs. 4-a 
and 4-b, we see that for a large range of binning boxes (as given by $b$ ), the apparent Renyi codimension $\overline{\mathcal{D}}_{q}$, is in fact $q$ - independent. This means that the $2 D$ and the horizontal intermittency behaviour is in fact described by a simple fractal structure. We read off the final Renyi codimensions

$$
\overline{\mathcal{D}}^{(2 D)}=0.023 \pm 0.004
$$

for the $2 D$ factorial moments and

$$
\overline{\mathcal{D}}^{(H)}=0.021 \pm 0.004
$$

for the horizontal factorial moments. In section 4 , we shall return to an interpretation of these results.

On the other hand, the data obtained from the vertical factorial moments show quite a distinct behaviour, see fig. 4-c. It is not possible to define anything like a single Renyi codimension. To our best knowlegde, this is the first example of a system displaying such a strong anisotropy in the factorial moments. We note that for the maximal values of $b$, the effective $\overline{\mathcal{D}}_{q}$ are close to the values obtained from the $2 D$ or the horizontal case. This is to be expected since for these very small boxes the effects of the anisotropy of the directed percolation clusters have not yet become apparent.

\section{Scaling relations and intermittency indices}

The features revealed by our numerical simulations and the estimates (3.1) and (3.2) are quite unexpected and thus require some theoretical interpretation. In particular, the obtained intermittency dimensions are much smaller (while non-zero) than the exponent ratios $\beta / \nu_{\|}, \beta / \nu_{\perp}$, (see (2.3)), contrary to some expectation in non-directed percolation [4]. Moreover, the similarity between $\overline{\mathcal{D}}^{(2 D)}$ and $\overline{\mathcal{D}}^{(H)}$ has to be explained. We shall now do so by using a simple scaling argument, eventually arriving at eq. (1.4).

Let us first consider the two-dimensional second moment:

$$
F_{2}^{(2 D)}:=<n(n-1)>/<n>^{2}
$$

where one keeps the simple normalization (1.1) which is sufficient for the theoretical discussion in the continuum limit. As already noticed in the literature [2, (1), the intermittency behaviour (1.2) is to be related to an 
effective singularity in the relevant correlation function. In the case of the 2-D moment, one may write:

$$
<n(n-1)>\underset{\delta \rightarrow 0}{\propto} \int_{[\delta]} d t_{1} d t_{2} d x_{1} d x_{2} G^{(2)}\left(x_{1}, x_{2} ; t_{1}, t_{2}\right)
$$

where $G^{(2)}$ is the two-point correlation function and the integral is over the phase-space box of unit length $\delta$, see fig. 2. This corresponds to the probability of a connecting path of occupied sites between the space-time points $\left(x_{1}, t_{1}\right)$ and $\left(x_{2}, t_{2}\right)$. Note an implicit averaging over phase-space boxes which is allowed by translational invariance, well verified in our simulations. It is also important to notice that, for practical simulation purpose, the binsize $\delta$ has to be considered very small, but large with respect to the bond size.

The scaling behaviour of $G^{(2)}$ near the percolation transition point $p_{c}$ can be written:

$$
G^{(2)} \sim(\delta p)^{2 \beta} \Psi\left[(\delta p)^{\nu_{\perp}} \theta ;(\delta p)^{\nu_{\|}} \tau\right] \sim \theta^{-\frac{2 \beta}{\nu_{\perp}}} \cdot \text { cste }
$$

where $\delta p=p-p_{c}, \theta=\left|x_{1}-x_{2}\right|$ and $\tau=\left|t_{1}-t_{2}\right|$ and $\Psi$ is a scaling function.

The first part of relation (4.2) comes from the critical behaviour at the percolation transition while the second part is the dominant $\delta p$-independent behaviour after rescaling. Note that the known strong anisotropy in time of directed percolation provides a hint for the dominance of the $\theta$ singularity over the phase-space box. This typical feature of directed percolation is well demonstrated by the narrowness of percolation clusters in the "time" direction, see ref. [8].Inserting the behaviour (4.2) in the estimate (4.1) at small $\delta$ one finds:

$$
<n(n-1)>\sim \delta^{4-2 \beta / \nu_{\perp}}
$$

the exponent 4 being simply interpreted as the naive integration dimension in (4.1).

The key remark of our analysis now coming is related to the normalization denominator $<n>^{2}$ of $F_{2}^{(2 D)}$. An oversimplified evaluation of this denominator would have led to some number proportional to the phase-space, namely $\delta^{4}$, and thus to an intermittency exponent $\beta / \nu_{\perp}$. This is much too large as compared with the numerical value (3.1). Rather, one has to take into account the density of occupied sites in the box $[\delta]$, which is not uniform. This density is better described by a fractal set whose dimension $D$ has been 
recently numerically estimated from the formation of very long percolation clusters [8]. Using this information, we write:

$$
<n>\sim \delta^{D}=\delta^{2-\bar{D}}
$$

introducing the fractal dimension of occupied sites and its codimension $\bar{D}$. We thus get the general relation:

$$
F_{2}^{(2 D)} \sim\left(\delta^{-2}\right)^{\left(\beta / \nu_{\perp}-\bar{D}\right)}
$$

Comparing with (1.3), we note that since the boxes considered are twodimensional, we have $F_{q}^{(2 D)} \sim\left(\delta^{-2}\right)^{\phi_{q}}$. In terms of the Renyi codimensions $\overline{\mathcal{D}}_{q}^{(2 D)}$ of a (fractal) intermittent process, we recover indeed the relation (1.4). The factorial moment satisfies

$$
F_{2}^{(2 D)}:=\frac{<n(n-1)>}{<n>^{2}} \sim \frac{<\rho^{2}>}{<\rho\rangle^{2}} \geq 1
$$

which implies in turn that the Renyi codimension $\overline{\mathcal{D}}$ is non-negative. From our numerical estimate (3.1), we see that the fractal cluster codimension $\bar{D}$ and the exponent ratio $\beta / \nu_{\perp}$ are very close, up to a small difference of definite sign.

It is of interest to compare this result with earlier determinations of the fractal dimension of directed percolation clusters. A careful study of this question was presented in [8]. Three possibilities to calculate $\bar{D}$ were considered. The first one simply assumes that the two-dimensional fractal can be considered as a direct product. The second possibility relies on a deterministic growth argument. Finally, the third possibility proceeds along a scaling argument taking into account the presence of the two length scales given by $\xi_{\|}$and $\xi_{\perp}$ [19. These arguments lead to 8

$$
\bar{D}^{(2 D)}=\left\{\begin{aligned}
\beta\left(\frac{1}{\nu_{\|}}+\frac{1}{\nu_{\perp}}\right) & \simeq 0.412 & & \text { direct product } \\
2 \beta /\left(\nu_{\|}+\nu_{\perp}\right) & \simeq 0.196 & & \text { deterministic growth } \\
\beta / \nu_{\perp} & \simeq 0.253 & & \text { scaling [19] }
\end{aligned}\right.
$$

Via (1.4), this would lead to $\overline{\mathcal{D}}^{(2 D)} \simeq-0.16,0.06$ and 0 , respectively. A precise numerical calculation using box-counting arguments yields [8]

$$
D=1.765 \pm 0.005
$$


and one notes a discrepancy with all of the relations (4.7).

However, (4.8) would yield via (1.4) a value $\overline{\mathcal{D}}^{(2 D)}=0.018(5)$ in agreement with our estimate (3.1). In other terms, one may interpret the small but non-zero difference between the cluster and correlation fractal dimensions as due to a weak intermittency phenomenon in $1+1$-dimensional directed percolation.

The same relation (1.4) holds for the "space" moments $F_{2}^{(H)}$, but it requires a more refined way of derivation, since the 2-point correlation function is zero between 2 sites with no "time" separation, see fig. 2 . In this particular case, one has to consider a 3-point correlation function $G^{(3)}$ linking the two given sites $\left(x_{1}, t_{1}\right)$ and $\left(x_{2}, t_{2}\right)$, starting from an initial $\left(x_{0}, t_{0}\right)$. One would assume the scaling behaviour

$$
G^{(3)} \sim(\delta p)^{3 \beta} \Phi\left[(\delta p)^{\nu_{\perp}} \theta_{i j} ;(\delta p)^{\nu_{\|}} \tau_{i j}\right] \sim \theta_{12}^{-\frac{3 \beta}{\nu_{\perp}}} \cdot \text { cste }
$$

where $\delta p=p-p_{c}, \theta_{i j}=\left|x_{i}-x_{j}\right|$ and $\tau_{i j}=\left|t_{i}-t_{j}\right|$ and $i, j=0,1,2 ; i \neq j$. Integrating over the initial conditions at $\left(x_{0}, t_{0}\right)$, and the phase-space box of linear dimension $\delta$ for $\left(x_{1}, t_{1}\right)$ and $\left(x_{2}, t_{1}\right)$ (note that we are at equal time), one gets:

$$
\begin{aligned}
<n(n-1)> & \sim \delta^{D_{\perp}+1-\beta / \nu_{\perp}} \\
<n> & \sim \delta^{D_{\perp}} \\
F_{2}^{(H)} & \sim \delta^{-\left(\beta / \nu_{\perp}-\bar{D}_{\perp}\right)}
\end{aligned}
$$

where $D_{\perp}$ is the "horizontal" fractal dimension. One finds again the same type of relation (1.4), which thus appears quite general.

We also note that our numerical estimates (3.1) and (3.2) suggest that the fractal codimensions $\bar{D}^{(2 D)} \simeq \bar{D}_{\perp}$, which is in agreement with the literature [19, 8].

A simple relation as (1.4) would have to be modified in the case of the "time" dimension, due to the multifractal character manifested by our numerical simulation. It is to be noticed that such a feature is in general associated with non-equilibrium cascading processes [20]. It is thus to our knowledge, the first time that multifractal fluctuations appear in a percolation problem, in probable relation with the oriented "time" direction characteristic of directed percolation. 


\section{Conclusions and outlook}

Let us summarize our results:

1. On the basis of a simulation of directed bond percolation in 1-" space"-1"time" dimensions and after checking against statistical and boundary effects the stability of our results, we have found that intermittency patterns of fluctuations show up in the distribution of occupied sites clusters. The fluctuations along the "space" dimension and in two dimensions are dominated by weak, fractal, structures of Renyi codimension $\overline{\mathcal{D}}=0.022 \pm 0.004$, (the mean value of (3.1) and (3.2)) while "time"-like fluctuations appear with a more complicated structure of multi-fractal type.

2. These numerical results can be interpreted as resulting from the scaling behaviour of the two- and three-point correlation function in the continuum limit at the bond percolation critical point, both corrected by the fractal dimension of clusters. The overall result is summarized in formula (1.4), which appears well verified for the two recognized fractal cases, "spatial" and two-dimensional. These two cases were seen to have the same intermittency indices. Our result clarifies a discussion on the calculation of fractal dimensions of directed percolation in the literature 8$]$.

It is quite interesting to note that, in its generalized form (1.4), the relation expressing the intermittency indices (in the fractal case) as a function of the correlation indices and the fractal cluster dimension can be applied to other problems. Let us mention the case of percolation at a second-order phase transition [3], which is also fractal, according to general scaling arguments [4]. An interesting example is the two-dimensional Ising spin system, where the relation (1.4) leads to the following prediction:

$$
\overline{\mathcal{D}}=\beta / \nu-\bar{D}=7 / 96
$$

where the exponent ratio $\beta / \nu=1 / 8$ is well known and the value of the fractal codimension $\bar{D}=5 / 96$ can be obtained by conformal invariance techniques [21]. The result (5.1), which is in agreement with numerical simulations [18], and finite-size scaling evaluations [22], clarifies a set of contradicting issues 
on the subject. While different from the value $\overline{\mathcal{D}}=\beta / \nu$, predicted in ref. [ [⿴囗十, the value (5.1) is different from the fractal dimension $\bar{D}$ of clusters as well. Indeed, the system admits a set of fractal fluctuations around a fractal object, which here was the average percolation cluster. We also remark that the value found in 22] is not accidental, as conjectured by the authors, but a prediction valid at the continuum limit.

Finally, the access to so refined quantities as fluctuation moments allowed by numerical calculations of directed percolation is an incentive to perform the same analysis in $2+1$ dimensions, with the bonus of a direct application to particle physics through the Reggeon Field model. Note that the already obtained result in $1+1$ dimension, applied to the Swinger-type model [5, 6], confirm the existence of weak intermittent fluctuations, seen in the model simulations [5].

\section{Acknowledgements}

We acknowledge R. Bidaux for a valuable discussion and Y. Leroyer for a careful reading of the manuscript. The present work was initiated at CERN (Theory Division) and Geneva University. One of us (MH) has the pleasure to thank the Service de Physique Théorique at CEA Saclay for its warm hospitality while this work was finished. 


\section{Figure captions}

Figure 1: The evolution as described by directed bond percolation. The result of a simulation on a small lattice with 32 "spatial" sites and periodic boundary conditions is shown. The triangles show some typical evolution cells whose conditional probabilities are recalled below the simulation sample. Note that, by convention, white dots are for empty sites and stars for occupied ones.

Figure 2: Binning for the definition of the factorial moments. Typical binning boxes are represented on the simulated patterns of sites (same series as Fig.1): Two-dimensional, Horizontal and Vertical examples are shown, together with typical connected paths which contribute to the singular behaviour at the continuum limit (see text).

Figure 3: Effective Renyi codimension $\overline{\mathcal{D}}_{2}$ as obtained from the factorial moment $(\mathrm{a}) F_{4}^{(2 D)}$, (b) $F_{4}^{(H)}$, (c) $F_{4}^{(V)}$ as function of the binning size $L / 2^{b}$. The curves corresponds to the following lattices $\bigcirc: N=8192, L=2048$ periodic boundary conditions (full curve), $\bigcirc: N=8192, L=2048$ free boundary conditions (dotted), $\diamond: N=4096, L=1024$ free boundary conditions (dashed) and $\square: N=4096, L=4096$ periodic boundary conditions (dashdotted). Notice the quite larger vertical coordinate scale in fig.(c).

Figure 4: Renyi codimensions $\overline{\mathcal{D}}_{q}=\phi_{q} /(q-1)$ as obtained for $N=8192$ sites, a maximal box size of $L=2048$ and periodic boundary conditions as obtained from the factorial moments (a) $F_{q}^{(2 D)}$, (b) $F_{q}^{(H)}$, (c) $F_{q}^{(V)}$. The symbols correspond to the following values of q $\bigcirc q=2, \bigcirc q=3, \diamond q=4$, $q=5$ and $\square q=6$. Notice the quite larger vertical coordinate scale in fig.(c). 


\section{References}

[1] Y. Pomeau Physica 23D, 3 (1986)

[2] For a recent review, see A. Bialas Nucl. Phys. A525, 345c (1991)

[3] J. Wosiek Acta Physica Polon. B19, 863 (1988)

[4] H. Satz Nucl. Phys. B326, 613 (1989)

[5] A. Bialas, W. Czyz, A. Dyrek, W. Florkowski and R. Peschanski Phys. Lett. 229B, 398 (1989)

[6] V.D. Barger and R.J.N. Philips Collider Physics (Addison-Wesley 1987), p. 183

[7] A. Bialas and R. Peschanski Nucl. Phys. B273, 703 (1986); B308, 857 (1988)

[8] B. Hede, J. Kertész and T. Vicsek J. Stat. Phys. 64, 829 (1991)

[9] J.L. Cardy and R.L. Sugar J. Phys. A13, L423 (1980)

[10] for a review, see W.Kinzel in Percolation Structures and Processes, Ann. Isr. Phys. Soc. Vol. 5, edited by G.Deutscher, R. Zallen and J. Adler (Bar-Ilan University 1983), p. 425

[11] J.W. Essam, A.J. Guttmann and K. De' Bell J. Phys A21, 3815 (1988)

[12] J. Benzoni J. Phys. A17, 2651 (1984)

[13] R. Brower, M.A. Furman and K. Subbarao Phys. Lett. 78B, 213 (1978)

[14] M. Henkel and H.J. Herrmann J. Phys. A23, 3719 (1990)

[15] P. Grassberger J. Phys. A22, 3673 (1989)

[16] H. Chaté and P. Manneville Physica 32D, 409 (1988)

[17] I. Novak, Bratislava Preprint (1989)

[18] S. Gupta, P. La Cock and H. Satz, Nucl. Phys. B362, 583 (1991) 
[19] J.P. Nadal, B. Derrida and J. Vannimenus Phys. Rev. B30, 376 (1984)

[20] R. Peschanski Nucl. Phys. B327, 144 (1989)

[21] B. Duplantier Physica 38D, 71 (1989); B. Duplantier and H. Saleur Phys. Rev. Lett. 63, 2536 (1989); A. L. Stella and C. Vanderzande Phys. Rev. Lett. 62, 1067 (1989)

[22] Z. Burda, J. Wosiek and K. Zalewski Phys. Lett. bf 266B, 439 (1991) 